\title{
Expression of domain III of the envelope protein from GP-78: a Japanese encephalitis virus
}

\author{
Sahil Kulkarni ${ }^{1} \cdot$ Sandeepan Mukherjee $^{2} \cdot$ Krishna Mohan Padmanabha Das $^{3}$. \\ Kaushiki Prabhudesai $^{1}$ - Nupur Deshpande ${ }^{1} \cdot$ Sushant Karnik $^{1} \cdot$ Abhay S. Chowdhary $^{2}$. \\ Usha Padmanabhan ${ }^{4}[$
}

Received: 19 December 2016/ Accepted: 20 April 2017/Published online: 25 May 2017

(C) Indian Virological Society 2017

\begin{abstract}
Acute encephalitis caused by the Japanese encephalitis virus (JEV) represents a growing epidemic and is a cause for concern in Southeast Asia. JEV is transmitted to humans through the bite of the Culicine mosquito species. The virus genome comprising of an RNA strand also encodes the envelope protein (E) which surrounds the virus. The $\mathrm{E}$ protein aids in fusion of virus with the cellular membrane of the host cell with the help of three structurally distinct domains (DI, DII, DIII) that are connected by flexible hinge regions. Of these domains, DIII (JEVDIII) has been reported to interact with the cellular membrane, aid viral entry and viral replication. Hence JEV-DIII has the potential to be an antigen that can provide immune protection to a JEV infection. In this study, we describe the cloning and expression of DIII of GP-78, a virulent strain of JEV prevalent in India. Our data clearly shows that JEV-
\end{abstract}

Electronic supplementary material The online version of this article (doi:10.1007/s13337-017-0379-3) contains supplementary material, which is available to authorized users.

Usha Padmanabhan

u.padmanabhan@haffkineinstitute.org

1 Department of Zoonosis, Haffkine Institute for Training, Research and Testing, Acharya Donde Marg, Parel, Mumbai 400 012, India

2 Department of Virology, Haffkine Institute for Training, Research and Testing, Acharya Donde Marg, Parel, Mumbai 400 012, India

3 Institute of Molecular Bioscinces, Humboldstrasse 50/1, 8010 Graz, Austria

4 Department of Cell Biology, Haffkine Institute for Training, Research and Testing, Acharya Donde Marg, Parel, Mumbai 400 012, India
DIII expressed from pVAC1 in HEK293T cells is membrane targeted. To our knowledge, this is the first demonstration of a recombinant construct that may block JEV entry into the cells and/or evoke specific antibodies against JEV. Future studies will reveal if our construct will elicit significant immune responses which will alleviate or ameliorate the pro-inflammatory responses induced by JEV.

Keywords JEV · Envelope protein - Domain III

Japanese encephalitis along with Dengue fever, Yellow fever, West Nile encephalitis are diseases caused by viruses belonging to the flaviviridae family. JEV is predominantly transmitted to humans by the Culicine mosquitoes, of which Culex tritaeniorhyncus, Culex gelidus are the principal vectors $[4,17]$. More than 3 billion people are now living and over 70 million children are born in JE endemic regions each year $[4,34]$.

JEV is a small, enveloped icosahedral virus, in which the single-stranded, positive-sense RNA viral genome $(\sim 11 \mathrm{~kb})$ consists of single open reading frame (ORF) encoding a polyprotein that is processed into three structural (core-C, premembrane-pRM, and envelope-E) and seven nonstructural (NS1, NS2A, NS2B, NS3, NS4A, NS4B, and NS5) proteins, flanked by $5^{\prime}-$ and $3^{\prime}$-nontranslated regions (NTRs) [6]. The viral RNA is complexed to multiple copies of the capsid protein forming the nucleocapsid assembly [20]. The spherical nucleocapsid is surrounded by the envelope (E) protein, which is glycosylated. In mature virions, the $\mathrm{E}$ glycoproteins are arranged in 90 homodimers with sets of three $\mathrm{E}$ head-to-tail homodimers that lie in 30 rafts and form a her-ringbone pattern [16]. 
Flaviviruses like JEV utilize clathrin-mediated endocytosis for cell entry [14, 28]. The E protein plays important role in viral attachment, membrane fusion, and entry of virus. It is a major structural protein that contains numerous neutralization epitopes, which mediates attachment of virus to the host cells. Both poly and monoclonal antibodies inhibit viral attachment to host cells or block viral penetration by binding to these epitopes. For example, it contains one putative $\mathrm{N}$ linked glycosylation site at N154 or a putative receptor-binding domain that induces the host immune response. Immunogenicity of DNA vaccine synthesizing the two forms of $E$ protein i.e. secretory and membrane anchored was tested in mice using intradermal and intramuscular delivery. Approximately $60 \%$ protection was observed to be provided by the vaccine irrespective of the route of vaccine delivery and form of E protein [7, 18]. In order to localize the neutralization antigenic site in the linear amino acid sequence of JEV E protein, Mason et al. [25] synthesized the small fragments of the E protein as trpE fusion proteins in E. coli. They found that E protein segment containing residues 303-396 was the shortest sequence capable of reacting with various JEV-neutralizing monoclonal anti-bodies $[15,31]$. Several authors have used the Envelope protein of viruses as the immune response provoking agent (for examples, see [10, 27]).

The E protein ectodomain has three structurally distinct domains (DI, DII, DIII) that are connected by flexible hinge regions [30]. The E protein exits as a homodimer in which each monomer has three $\beta$-barrel domains viz. domain I, domain II and domain III. Among these domains, Domain III is an immunoglobulin (Ig)-like domain that is thought to contain the putative receptor-binding sites 32-37 [26]. Negatively charged glycoaminoglycans, such as heparan sulfate, which are abundantly ex-pressed on numerous cell types are utilized as low-affinity attachment factors by several flavi-viruses. These interactions serve to concentrate the virus at the cell surface and are mediated by domain DIII of the E glycoprotein. DIII also plays a vital role in internalization of the virus and docking with cellular membranes to aid viral replication. JEV-DIII has been shown to be both immune reactive and immune response provoking by several authors [2, 19, 33]. Thus we hypothesized that the DIII could be a potential antigen for future vaccine development and we studied the localization of DIII in mammalian cells. In the present study, we report the cloning and expression of Domain III (DIII) of the Envelope protein (E) of GP-78, a virulent strain of JEV prevalent in India in mammalian cells.

The WHO prequalified JEV vaccine, SA-14-14-2 is a live attenuated virus which does not cross the blood-brain barrier but evokes similar immune responses in the host. SA-14-14-2 has three major problems: (1) It is not suited for infant's below 1 years of age, (2) There is a requirement for booster doses to achieve sustainable immunity and (3) Patient is to be kept under observation for $48 \mathrm{~h}$ to 1 week after administration of vaccine as many side effects are observed [3, 11, 13].

In this regard, DNA vaccines provide an alternative to conventional vaccines and have achieved success against several families of viruses such as HIV [32] HBV [9], Influenza virus etc. [23]. DNA vaccines are plasmids encoding a gene of interest that can be propagated in bacteria so that the gene of interest is expressed in mammalian cells (by means of a strong viral promoter) and provokes a good immune response conferring immunity against the particular pathogen [8, 21]. This line of approach is also emerging for vaccine development against JEV and the envelope protein (E) has been the target of many studies.

Given that JEV-DIII has been shown to be both immune reactive and immune response provoking by several authors [2, 19, 33]. Thus we hypothesized that the DIII could be a potential antigen for future vaccine development and we studied the localization of DIII in mammalian cells. In the present study, we report the cloning and expression of Domain III (DIII) of the Envelope protein (E) of GP-78, a virulent strain of JEV prevalent in India in mammalian cells.

The JEV strain GP78 (JEV-GP78), which was responsible for the epidemic in Gorakhpur, India in 1978, is still the most prevalent strain in India and was kindly provided by Dr. T.N. Dhole, Sanjay Gandhi Post-Graduate Institute (SGPGI), Lucknow, India. Virus was amplified by injecting quarantined suckling mice with the virulent strain $(0.1 \mathrm{ml})$ of JEV intracerebrally and observation of associated morbidity in mice such as hind limb paralysis, hunching and ruffling. Post-observation of symptoms, mice were sacrificed, brains harvested and homogenized in phosphate buffered saline (PBS). The homogenates were centrifuged at $5000 \mathrm{rpm}$ for $10 \mathrm{~min}$ at $4{ }^{\circ} \mathrm{C}$ and the supernatant was used as the viral stock for further experiments. All viral stocks were stored at $-80{ }^{\circ} \mathrm{C}$ until further use.

The envelope (E) protein of the SA-14-14-2 genome (Genbank number U14163.1), spans from 978-2478 bp consisting of 500 amino acids of the viral protein of which 111 amino acids, from 291 to 402 has been designated as Domain III of the E protein (JEV-DIII) [19]. The JEV-DIII peptide corresponds to a RNA/DNA to a fragment of length 333 bp (1851-2184 bp, see Supp Fig 1). We chose to clone and express DIII because (1) recombinant expression of DIII as an isolated peptide, would compete with JEV and prevent cellular entry of the virus and (2) ectopic expression of DIII may also become antigenic and provoke an immune response against JEV in vivo. However since the vaccine strain of JEV, SA-14-14-2 is a mutated virus which does not enter the brain, we cloned 


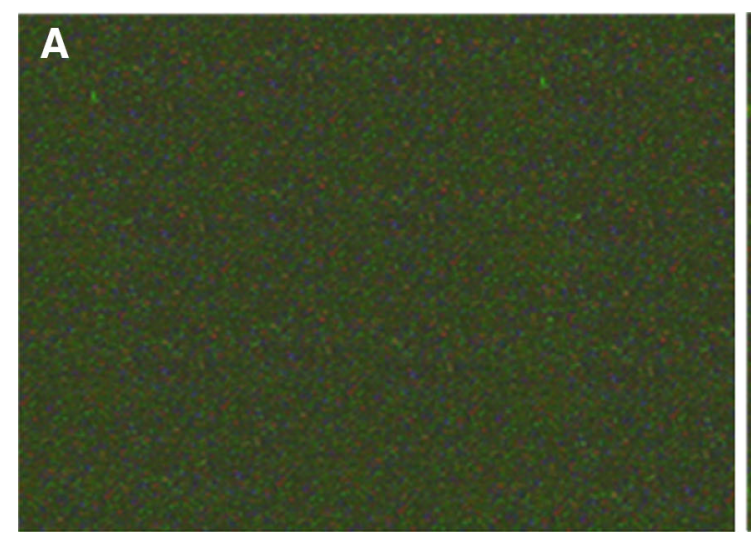

Fig. 1 GFP-DIII localizes to the membrane in HEK293-T cells. JEVDIII was cloned into the plasmid pVAC and was transfected into HEK293T cells by the $\mathrm{CaCl}_{2}$ method. JEV was visualized by using a specific antibody for DIII and fluorescence microscopy. a Shows the

and expressed DIII of GP-78 (the amino acid numbers are same to that mentioned for SA-14-14-2). To aid cloning into a variety of plasmids, JEV-DIII was first cloned into a cloning vector pJET-III as per manufacturer's instructions by blunt ligation. Ligation products were transformed into E. coli (DH5a). Colonies were screened for the presence of the recombinant pJET-JEV-DIII by colony PCR (Supp Fig 1A). Plasmid DNA was isolated and the purified plasmids were sent for sequencing using both forward and reverse primers. Sequencing results indicated the presence of positive clones containing recombinant pJET-JEV-DIII (Supp Fig 1B). Translation of the sequencing results and alignment reveals the cloned DIII contains $\mathrm{G}$ rather than $\mathrm{E}$ at position 88 similar to that of GP78 but not similar to that of SA-14-14-2 (Supp 1C).

pVAC1 (InvivoGen) is a plasmid designed for preparation of DNA vaccines. The vector was designed so that any protein expressed from this plasmid is expressed at the cell membrane because of the presence of a glycophosphatidylinositol (GPI) anchoring domain of human PLAP which acts as membrane localization signal [1]. The PLAP signal peptide consists of the $C$ terminal amino acids of PLAP which is bound to the membrane by a phosphatidylinositol anchor and is cleaved off during signaling [1]. The PLAP signal peptide has also been used by other authors to enable membrane localization and secretion of peptides [5].

Further pVAC1 is also a muscle targeting vector as the backbone and comparison of routes of delivery of plasmids into rodents or humans have revealed that intramuscular delivery of the DNA yield the highest titer values [24]. In addition, vectors encoding IL-2 had enhanced humoral and cellular immune responses to an exogenous antigen $[12,22,29]$. Thus we expect that the PLAP and the IL-2 signals in pVAC1 will target and anchor the JEV domain

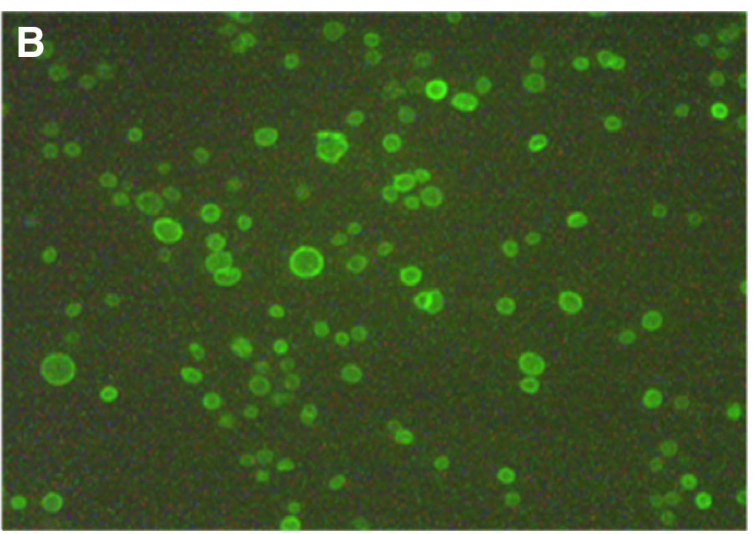

immunohistochemical analysis of cells transfected with the empty vector control. b Shows the immunohistochemical analysis of cells transfected with recombinant pVAC-JEV-DIII

III to the muscle membrane for immune processing, assist secretion of the antigen and will provoke a humoral response.

To clone JEV-DIII into pVAC1, the recombinant pJETJEV-DIII vector was digested with EcoR1 and BamH1, the fragment isolated and ligated with a EcoR1-BamH1 digested pVAC1 to yield the recombinant pVAC1-JEVDIII and positive colonies using $\mathrm{pVAC} 1$ specific primers (Supp Fig 2B).

After confirmation by sequencing, recombinant $\mathrm{pVAC} 1$ containing JEV-DIII-GFP was isolated and transfected into HEK293T cells (Fig. 1a, b). As can be seen DIII localizes to the membranes of HEK cells. Our data clearly shows that JEV-DIII expressed from pVAC1 in HEK293T cells is membrane targeted (Fig. 1b). To our knowledge, our study is the first such attempt to create a recombinant construct which may through virtue of DIII expression on the membrane prevent JEV entry and could also provoke an antibody response. This is not known at present and has to be investigated.

Acknowledgements The authors thank the Indian Council of Medical Research (ICMR), New Delhi for providing senior research fellowship to Sahil Kulkarni (File No-BMS/IMM/67/06/2012). We would also like to thank staff and students of the Department of Virology and Zoonosis for their helpful discussions and support. We are grateful to Dr. Hemant Kawalkar, D.Y. Patil School of Biotechnology, Dr. Murali Chilkapatti and Dr. Vaidya from ACTREC, Mumbai for help with experiments.

\section{References}

1. Bailey CA, Gerber L, Howard AD, Udenfriend S. Processing at the carboxyl terminus of nascent placental alkaline phosphatase in a cell-free system: evidence for specific cleavage of a signal peptide. Proc Natl Acad Sci. 1989;86(1):22-6. 
2. Bharati K, Malik Y, Vrati S. Immunogenicity and protective efficacy of the E. coli-expressed domain III of Japanese encephalitis virus envelope protein in mice. Med Microbiol Immunol. 2007;196(4):227-31.

3. Bista MB, Banerjee M, Shin SH, Tandan J, Kim MH, Sohn YM, et al. Efficacy of single-dose SA 14-14-2 vaccine against Japanese encephalitis: a case control study. Lancet. 2001;358(9284):791-5.

4. Campbell GL, Hills SL, Fischer M, Jacobson JA, Hoke CH, Hombach JM, et al. Estimated global incidence of Japanese encephalitis: a systematic review. Bull World Health Organ. 2011;89(10):766-74.

5. Chen H, Leder P. A new signal sequence trap using alkaline phosphatase as a reporter. Nucleic Acids Res. 1999;27(4):1219-22.

6. Chen C-J, Kuo M-D, Chien L-J, Hsu S-L, Wang Y-M, Lin J-H. RNA-protein interactions: involvement of NS3, NS5, and 3'noncoding regions of Japanese encephalitis virus genomic RNA. J Virol. 1997;71(5):3466-73.

7. Chen H-W, Pan C-H, Liau M-Y, Jou R, Tsai C-J, Wu H-J, et al. Screening of protective antigens of Japanese encephalitis virus by DNA immunization: a comparative study with conventional viral vaccines. J Virol. 1999;73(12):10137-45.

8. Corr M, von Damm A, Lee DJ, Tighe H. In vivo priming by DNA injection occurs predominantly by antigen transfer. J Immunol. 1999;163(9):4721-7.

9. Davis HL, Mancini M, Michel M-L, Whalen RG. DNA-mediated immunization to hepatitis B surface antigen: longevity of primary response and effect of boost. Vaccine. 1996;14(9):910-5.

10. Forns X, Payette PJ, Ma X, Satterfield W, Eder G, Mushahwar $\mathrm{IK}$, et al. Vaccination of chimpanzees with plasmid DNA encoding the hepatitis $\mathrm{C}$ virus (HCV) envelope E2 protein modified the infection after challenge with homologous monoclonal HCV. Hepatology. 2000;32(3):618-25.

11. Ganesan VBV, Charles J, Alagappan U. A study on efficacy and safety of SA-14-14-2 vaccine against JapaneseEncephalitis in Virudhunagar district. Int J Res Health Sci. 2014 Jan31;1;2(1):111-7.(2(1)):111-7.

12. He X, Tsang TC, Zhang T, Luo P, Harris DT. Antigen epitopeexpressing cytokines for DNA immunization. Vaccine. 2005;23(16):1966-72.

13. Hoke CH, Nisalak A, Sangawhipa N, Jatanasen S, Laorakapongse $\mathrm{T}$, Innis BL, et al. Protection against Japanese encephalitis by inactivated vaccines. N Engl J Med. 1988;319(10):608-14.

14. Ishak R, Tovey D, Howard C. Morphogenesis of yellow fever virus 17D in infected cell cultures. J Gen Virol. 1988;69(2):325-35.

15. Kaur R, Vrati S. Development of a recombinant vaccine against Japanese encephalitis. J Neurovirol. 2003;9(4):421-31. doi:10. 1080/13550280390218454.

16. Kuhn RJ, Zhang W, Rossmann MG, Pletnev SV, Corver J, Lenches E, et al. Structure of dengue virus: implications for flavivirus organization, maturation, and fusion. Cell. 2002;108(5):717-25.

17. Kulkarni A, Sangar V, Kothari S, Mehta S, Dahake R, Mukherjee $\mathrm{S}$, et al. Construction of envelope domain III based recombinant tetravalent dengue vaccine. Int $\mathrm{J}$ Pharm Sci Rev Res. 2014;26(2):44.
18. Li J, Chen H, Wu N, Fan D, Liang G, Gao N, et al. Characterization of immune responses induced by inactivated, live attenuated and DNA vaccines against Japanese encephalitis virus in mice. Vaccine. 2013;31(38):4136-42. doi:10.1016/j.vaccine. 2013.06.099.

19. Lin C-W, Wu S-C. A functional epitope determinant on domain III of the Japanese encephalitis virus envelope protein interacted with neutralizing-antibody combining sites. J Virol. 2003;77(4):2600-6.

20. Lindenbach BD, Rice CM. Molecular biology of flaviviruses. Adv Virus Res. 2003;59:23-61.

21. Liu M. DNA vaccines: a review. J Intern Med. 2003;253(4):402-10.

22. Ma D, Gao M, Li J, Ma C, Li G. Construction of novel cytokine by fusion of chicken IL-2 signal peptide to mature chicken IL-15 and comparison of the adjuvant effects by DNA immunization against Eimeria challenge. Vet Immunol Immunopathol. 2013;156(1-2):114-20. doi:10.1016/j.vetimm.2013.09.005.

23. Macklin $M$, et al. Immunization of pigs with a particlemediated DNA-vaccine to infuenza A virus protects against challenge with homologous virus. J Virol. 1998;72:1491-6.

24. McCluskie MJ, Millan CB, Gramzinski RA, Robinson HL, Santoro JC, Fuller JT, et al. Route and method of delivery of DNA vaccine influence immune responses in mice and non-human primates. Mol Med. 1999;5(5):287.

25. Mason, P. W., Dalrymple, J. M., Gentry, M. K., McCown, J. M., Hoke, C. H., Burke, D. S., Mason, T. L. Molecular characterization of a neutralizing domain of the Japanese encephalitis virus structural glycoprotein. Journal of general virology. (1989); 70(8):2037-2049

26. Mukhopadhyay S, Kuhn RJ, Rossmann MG. A structural perspective of the flavivirus life cycle. Nat Rev Microbiol. 2005;3(1):13-22.

27. Neirynck S, Deroo T, Saelens X, Vanlandschoot P, Jou WM, Fiers W. A universal influenza A vaccine based on the extracellular domain of the M2 protein. Nat Med. 1999;5(10):1157-63.

28. Ng ML, Lau LC. Possible involvement of receptors in the entry of Kunjin virus into Vero cells. Adv Virol. 1988;100(3):199-211.

29. Raz E, Watanabe A, Baird SM, Eisenberg RA, Parr TB, Lotz M, et al. Systemic immunological effects of cytokine genes injected into skeletal muscle. Proc Natl Acad Sci. 1993;90(10):4523-7.

30. Rey FA, Heinz FX, Mandl C, Kunz C, Harrison SC. The envelope glycoprotein from tick-borne encephalitis virus at 2 angstrom resolution. Nature. 1995;375(6529):291.

31. Tiwari S, Singh RK, Tiwari R, Dhole TN. Japanese encephalitis: a review of the Indian perspective. Braz $\mathrm{J}$ Infect Dis. 2012;16:564-73.

32. Ugen KE, Nyland SB, Boyer JD, Vidal C, Lera L, Rasheid S, et al. DNA vaccination with HIV-1 expressing constructs elicits immune responses in humans. Vaccine. 1998;16(19):1818-21.

33. Wu S-C, Yu C-H, Lin C-W, Chu I-M. The domain III fragment of Japanese encephalitis virus envelope protein: mouse immunogenicity and liposome adjuvanticity. Vaccine. 2003;21(19):2516-22.

34. Yun S-I, Lee Y-M. Japanese encephalitis: the virus and vaccines. Hum Vaccin Immunother. 2014;10(2):263-79. 